Check for updates

Cite this: Phys. Chem. Chem. Phys., 2021, 23, 5615
Received 18th December 2020, Accepted 21st February 2021

DOI: $10.1039 / \mathrm{d} 0 \mathrm{cp} 06553 f$

rsc.li/pccp

\section{Site-by-site tracking of signal transduction in an azidophenylalanine-labeled bacteriophytochrome with step-scan FTIR spectroscopy $\dagger$}

\author{
Moona Kurttila, (DD a Brigitte Stucki-Buchli, ${ }^{a}$ Jessica Rumfeldt, ${ }^{a}$ Lea Schroeder, ${ }^{b}$ \\ Heikki Häkkänen, ${ }^{a}$ Alli Liukkonen, ${ }^{a}$ Heikki Takala, (D) ${ }^{a}$ Tilman Kottke (D) *b and \\ Janne A. Ihalainen (D) *a
}

\begin{abstract}
Signal propagation in photosensory proteins is a complex and multidimensional event. Unraveling such mechanisms site-specifically in real time is an eligible but a challenging goal. Here, we elucidate the site-specific events in a red-light sensing phytochrome using the unnatural amino acid azidophenylalanine, vibrationally distinguishable from all other protein signals. In canonical phytochromes, signal transduction starts with isomerization of an excited bilin chromophore, initiating a multitude of processes in the photosensory unit of the protein, which eventually control the biochemical activity of the output domain, nanometers away from the chromophore. By implementing the label in prime protein locations and running two-color step-scan FTIR spectroscopy on the Deinococcus radiodurans bacteriophytochrome, we track the signal propagation at three specific sites in the photosensory unit. We show that a structurally switchable hairpin extension, a so-called tongue region, responds to the photoconversion already in microseconds and finalizes its structural changes concomitant with the chromophore, in milliseconds. In contrast, kinetics from the other two label positions indicate that the site-specific changes deviate from the chromophore actions, even though the labels locate in the chromophore vicinity. Several other sites for labeling resulted in impaired photoswitching, low structural stability, or no changes in the difference spectrum, which provides additional information on the inner dynamics of the photosensory unit. Our work enlightens the multidimensionality of the structural changes of proteins under action. The study also shows that the signaling mechanism of phytochromes is accessible in a time-resolved and site-specific approach by azido probes and demonstrates challenges in using these labels.
\end{abstract}

\section{Introduction}

A family of photoreceptors called phytochromes mediate red and far-red light responses in plants, bacteria, algae, and fungi. These proteins undergo photoinduced structural changes which lead to biochemical signaling cascades that regulate the growth and development according to the ambient conditions. ${ }^{1-3}$ Phytochromes cycle between two metastable states, the red light-absorbing Pr state and the far-red lightabsorbing Pfr state. In canonical phytochromes, the photoconversion from the Pr state to the Pfr state is triggered by the isomerization of the covalently bound bilin chromophore,

\footnotetext{
${ }^{a}$ University of Jyväskylä, Nanoscience Center, Department of Biological and Environmental Science, 40014 Jyväskylä, Finland. E-mail: janne.ihalainen@jyu.fi

${ }^{b}$ Physical and Biophysical Chemistry, Department of Chemistry,

Bielefeld University, Universitätsstr. 25, 33615 Bielefeld, Germany.

E-mail: tilman.kottke@uni-bielefeld.de

$\dagger$ Electronic supplementary information (ESI) available. See DOI: 10.1039/ d0cp06553f
}

which leads to structural reorganization of the photosensory unit that eventually controls the activity of the output domain (Fig. 1A). The photoconversion proceeds from Pr to Pfr via at least two intermediate states, Lumi-R and Meta-R, respectively, ${ }^{4,5}$ and the protein returns to the Pr state by far-red light excitation (Fig. 1B). Alternatively, canonical phytochromes thermally revert back to the Pr state in the dark within minutes to hours. ${ }^{1}$

The conserved N-terminal photosensory module (PSM) of bacterial phytochromes consists of three domains, PAS (Period/ Arnt/Single-minded), GAF (cGMP phosphodiesterase/adenylate cyclase/FhlA) and PHY (phytochrome-specific) (Fig. 1A). The PHY domain is followed by a C-terminal output domain which in bacteriophytochromes is often a histidine kinase (HK). PAS and GAF together bind a biliverdin IX $\alpha(\mathrm{BV})$ as a chromophore. The four-ring (A-D) BV is covalently bound to the PAS domain via a thioether linkage between the A-ring and a cysteine residue. ${ }^{6}$ The non-covalent interactions with the GAF residues and a so-called pyrrole water further position the BV., ${ }^{6,7}$ The photo excitation of the $\mathrm{BV}$ chromophore causes isomerization of 

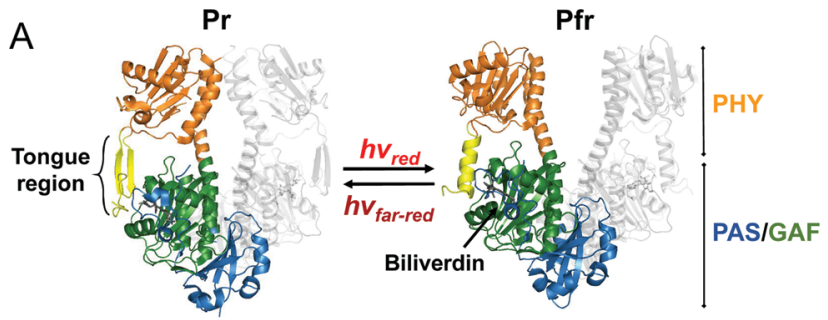

B
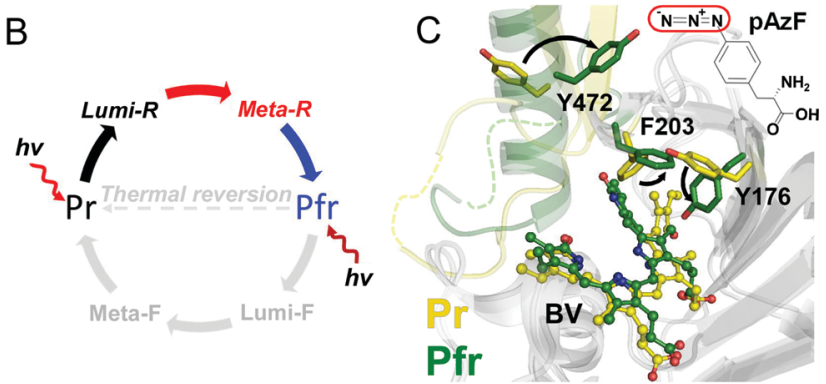

Fig. 1 Structure and photocycle of DrBphP PSM. (A) Phytochromes interconvert between red light-absorbing $\mathrm{Pr}$ and far-red light-absorbing Pfr states (PDB codes 4OOP and $5 \mathrm{C} 5 \mathrm{~K}$, respectively). The different domains of the photosensory module are indicated with different colors. The tongue region is indicated in yellow. (B) The photocycle of canonical phytochromes has at least two spectrally distinguishable intermediate states, Lumi-R and Meta-R. (C) The unnatural amino acid p-azidophenylalanine (pAzF), incorporated into a protein, contains an azide group (red circle) of which the vibrational frequency is distinct from all other modes of protein and chromophore (biliverdin, BV, ball-and-stick). The three pAzF-exchanged side chains are shown with sticks in Pr (yellow) and in Pfr (green).

the double bond between carbons 15 and 16,,9 which is observed as a rotation of the D-ring. This isomerization, in turn, changes the interactions between the chromophore and the residues in the BV binding pocket. ${ }^{10}$ Although located in separate domains, BV is linked to a hairpin extension, a so-called tongue region, via an aspartic acid and a tyrosine, both located in the GAF domain. ${ }^{7,11}$ Most probably mediated by these interactions, the tongue region restructures from $\beta$-sheet to $\alpha$-helix during the photoconversion (Fig. 1A).$^{11}$ Eventually, these events lead to structural changes in the whole protein, and the reorganization of the output domains ultimately controls the chemical activity of the receptor.

Time-resolved structural information is necessary to follow the signal propagation processes in photosensory proteins. This increases our understanding of the functional properties of proteins, in general. In the field of phytochromes, studies using techniques such as X-ray crystallography ${ }^{6,7,11}$ and nuclear magnetic resonance (NMR) spectroscopy ${ }^{12,13}$ have successfully shed light on the structural changes observed in the PSM between Pr and Pfr states without time-resolved information, however. With X-ray scattering ${ }^{4,11}$ only large-scale structural information has been obtained with time scales of the whole photocycle. Using IR spectroscopy, both the response of the bilin chromophore and the response of the protein can be followed in solution on all time scales of the photocycle. However, a molecule with $N$ atoms has $3 N-6$ vibrational modes and accordingly the assignment of absorption bands to single functional groups becomes extremely challenging for a large molecule such as phytochrome. Some vibrational modes in the IR difference spectra of phytochromes have been assigned to functional groups of the chromophore or protein using techniques such as isotope exchange, ${ }^{14,15}$ chemical modification of the chromophore, ${ }^{14}$ mutagenesis, ${ }^{16}$ truncation $^{17}$ and computations. ${ }^{8,18}$ Still, challenges in assignments remain such as the overlap with other modes and frequency shifts by slight conformational changes of the protein and the chromophore. An intriguing solution for this dilemma is an azido IRprobe with distinguishable vibrational properties. The introduction of vibrational labels into the protein offers a possibility to gain site-specific resolution for structural and chemical changes of proteins. ${ }^{19-24}$

Azido probes have been successfully employed to study proteins previously, ${ }^{25-31}$ for example rhodopsin activation, ${ }^{19,32}$ protein dynamics and interaction ${ }^{33}$ and ligand binding. ${ }^{20,34}$ They can be introduced into proteins by the unnatural amino acid $p$-azidophenylalanine (pAzF) through protein expression using the amber codon suppression technology. ${ }^{26,35,36}$ Together, this technology and the small size of pAzF enable placing the label at surface-exposed and buried positions of the protein. pAzF carries an azido group whose asymmetric $\mathrm{N}_{3}$ stretch vibration absorbs at around $2100 \mathrm{~cm}^{-1}$, in a distinct region from all other protein modes. ${ }^{37}$ Its high extinction coefficient of about $610 \mathrm{M}^{-1} \mathrm{~cm}^{-1}$ makes it superior to many other vibrational probes such as popular cyano probes (with $\varepsilon=120-220 \mathrm{M}^{-1} \mathrm{~cm}^{-1}$ ). ${ }^{22,38-40}$ Most importantly, pAzF is sensitive to changes in its electrostatic environment, especially to the H-bonding strength. ${ }^{38-41}$ Therefore, the structural changes of the protein and chromophore are reflected as changes in the spectral position and shape of the pAzF signal as detected by FTIR spectroscopy.

Previously, pAzF has provided information in the ultrafast time scale on structural changes in two BLUF domains ${ }^{30}$ and in the tens of milliseconds to seconds time scale on channelrhodopsin pore hydration with rapid-scan FTIR. ${ }^{31}$ Here, we resolve structural changes in the protein microenvironment in the micro- to millisecond time scale, desirable as well for other photoreceptors, to our knowledge for the first time with this label. We introduce pAzF in various positions (Fig. S1, ESI $\dagger$ ) of a bacterial phytochrome photosensory module from Deinococcus radiodurans, hereafter DrBphP ${ }_{\mathrm{PSM}}$, to detect local structural changes in the protein with a site-by-site approach in a time-resolved manner using two-color step-scan FTIR spectroscopy. ${ }^{5,42}$ As a result, we address how and when the microenvironment changes in these sites during the photocycle. Furthermore, we report on the effect of the strong dipole present in the azidophenylalanine on the kinetics of the photocycle, which provides information about the active role of these sites for the signal propagation in the protein.

\section{Materials and methods}

\subsection{Protein expression and purification of the unlabeled DrBphP PSM monomer}

We chose to introduce the $\mathrm{pAzF}$ to the monomeric form of $\operatorname{DrBphP} \mathrm{PSM}_{\mathrm{PS}}$ to simplify the protein purification procedure. 
This monomeric DrBphP $P_{\mathrm{PSM}}$ contains the mutations F145S, L311E and L314E, which are located at the dimer interface and prevent dimerization. ${ }^{43,44}$ The unlabeled, (His) ${ }_{6}$-tagged and monomeric DrBphP $P_{\mathrm{PSM}}$ was expressed in Escherichia coli BL21(DE3) as described previously. ${ }^{44}$ Purification was performed as described in Ihalainen et $a l^{5}$

\subsection{Production of pAzF-labeled $\mathrm{DrBphP} \mathrm{PSM}_{\mathrm{PS}}$ monomers}

The amber codon suppression technology ${ }^{26}$ was used to introduce the unnatural amino acid pAzF into the phytochrome. We replaced the original amino acid encoding codon with the amber codon (TAG) using the QuikChange Lightning Multi Site-Directed Mutagenesis kit (Agilent Technologies) in nine positions, Y176, F203, R228, S257, Y263, Y307, F372, F469, and Y472, each in a separate pET21b(+) plasmid (Novagen). ${ }^{44}$

The plasmid with the TAG-mutated gene encoding monomeric DrBphP ${ }_{\mathrm{PSM}}$ was co-transformed with $\mathrm{pEVOL}^{\mathrm{pAzF}} \mathrm{F}^{36}$ (Addgene plasmid \# 31186) into E. coli BL21(DE3). The cells were grown in LB medium supplemented with $150 \mu \mathrm{g} \mathrm{mL} \mathrm{m}^{-1}$ ampicillin and $25 \mu \mathrm{g} \mathrm{mL} \mathrm{m}^{-1}$ chloramphenicol. $0.02 \%$ arabinose and $600 \mu \mathrm{g} \mathrm{mL}^{-1} \mathrm{H}$-4-Azido-Phe-OH (Bachem) was added when the $\mathrm{OD}_{600}$ reached 0.2. The expression of the phytochrome was induced with $1 \mathrm{mM}$ IPTG at an $\mathrm{OD}_{600}$ of 0.8 . The incubator was covered to protect reactive $\mathrm{pAzF}^{35}$ from light. Further purification steps were carried out by using dim light or green light to protect both, pAzF and BV.

The cells were resuspended in $20 \mathrm{mM}$ Tris, $50 \mathrm{mM} \mathrm{NaCl}$, pH 8 and lysed with an EmulsiFlex-C3 homogenizer (Avestin). pAzF is a potentially reactive amino acid, therefore the incubation time of the protein in the cell lysate was kept as short as possible. The apoprotein was purified using $\mathrm{Ni}^{2+}$-affinity chromatography (HisTrap FF crude, GE Healthcare) in a $20 \mathrm{mM}$ Tris buffer, $\mathrm{pH} 8,50 \mathrm{mM} \mathrm{NaCl}$ and eluted with an imidazole gradient. By the affinity chromatography, the protein containing a C-terminal (His) $)_{6}$-tag was separated from truncated protein in which the amber codon was interpreted as a stop codon.

The apoprotein was supplemented with biliverdin chromophore (Frontier Scientific). A $10 \times$ molar excess of BV hydrochloride (Frontier Scientific), dissolved in a small amount of dimethyl sulfoxide, was added to the purified protein. The mixture was incubated at $4{ }^{\circ} \mathrm{C}$ overnight. The protein with $\mathrm{BV}$ bound was then purified by size-exclusion chromatography (HiLoad 26/600 Superdex 200 prep grade, GE Healthcare) in $30 \mathrm{mM}$ Tris, pH 8.

The purified protein was concentrated with Amicon Ultra concentration tubes with a $10 \mathrm{kDa}$ cutoff (Merck-Millipore) to about $25-30 \mathrm{mg} \mathrm{mL^{-1 }}$, flash frozen in liquid nitrogen and stored at $-80{ }^{\circ} \mathrm{C}$.

\subsection{Steady-state FTIR spectroscopy}

The steady-state FTIR spectroscopy of the labeled and unlabeled samples was performed using a Nicolet Magna IR-760 FTIR spectrometer with XT-KBr beam splitter and Ever-Glo IR source. The measurements were conducted using 100 scans in the spectral range of $400-3500 \mathrm{~cm}^{-1}$ with a resolution of $2 \mathrm{~cm}^{-1}$. Sample preparation and light-induced difference spectroscopy were performed as described in Takala et al. ${ }^{45}$
Absorption spectra were measured in buffered $\mathrm{D}_{2} \mathrm{O}$ at pD 8.0. Concentrated $\mathrm{HCl}$ in $\mathrm{H}_{2} \mathrm{O}$ was used to adjust the $\mathrm{pH}$ of $30 \mathrm{mM}$ Tris in $\mathrm{D}_{2} \mathrm{O}$ to $\mathrm{pH}^{*} 7.6$ corresponding to $\mathrm{pD}$ 8.0. The buffer of the protein was exchanged using Zeba Spin desalting columns with a $7 \mathrm{kDa}$ MWCO (Thermo Scientific). In buffered $\mathrm{D}_{2} \mathrm{O}$, the protein was switched between Pr and Pfr by illumination using $660 \mathrm{~nm}$ and $775 \mathrm{~nm}$ LEDs, respectively, in 5 min intervals for 1 hour to enhance the H/D exchange in both states. ${ }^{5}$ After switching, the buffer was exchanged for a second time, concentrated to about 2-3 mM with Vivaspin $50010 \mathrm{kDa}$ centrifugal concentrators (Sartorius) and filtered with Amicon $0.22 \mu \mathrm{m}$ centrifugal filters (Merck-Millipore). The path length was set to $50 \mu \mathrm{m}$ by using a Teflon spacer. The combination of high concentration and high path length resulted in saturated amide I and II bands, but those were disregarded as the focus was on the signal in the label region.

\subsection{Time-resolved step-scan FTIR spectroscopy}

Two-color step-scan FTIR spectroscopy with a time resolution of $2.5 \mu \mathrm{s}$ was performed on an IFS 66v spectrometer (Bruker) as described in Ihalainen et al. ${ }^{5}$ The detection window was extended to $2256 \mathrm{~cm}^{-1}$ to include the label region by placing an LP-4500 filter (Spectrogon) and $\mathrm{CaF}_{2}$ windows in front of the detector. $^{24} 316$ discrete mirror positions were stepped and undersampling was applied for FT. The resulting spectral resolution was $8 \mathrm{~cm}^{-1}$. To increase the signal in the label region, the path length was adjusted to achieve an $A_{1650}>1.1$, while keeping the protein concentration the same as in the steady-state experiments to ensure a sufficient hydration of the sample. The resulting high water absorption in the amide I region caused a saturation in the region of 1610 to $1670 \mathrm{~cm}^{-1}$, which was therefore left out in the global analysis of the difference spectra. 15 experiments on F203pAzF, 8 experiments on Y176pAzF and 11 experiments on Y472pAzF were averaged with 8 coadditions each, while exchanging the sample after less than 8000 excitations. All experiments were performed at $20{ }^{\circ} \mathrm{C}$.

\subsection{Steady-state UV-vis spectroscopy}

The steady-state UV-vis difference spectra were measured with a Cary 8454 UV-vis spectrometer (Aligent Technologies) using $1.5 \mathrm{~mL}$ plastic, semi-micro cuvettes (Brandtech Scientific) with $10 \mathrm{~mm}$ path length and $500 \mu \mathrm{L}$ sample. The sample was diluted to about $0.3 \mathrm{mg} \mathrm{mL} \mathrm{m}^{-1}$. The initial spectrum was recorded on a sample kept in the dark, then the sample was switched multiple times between Pr and Pfr using $661 \mathrm{~nm}$ and $775 \mathrm{~nm}$ LEDs, while spectra were measured in between the switching. The illumination times were set to reach full photoconversion.

\subsection{Flash photolysis}

The sample with absorbance of $0.7-0.8$ at $700 \mathrm{~nm}$ in a $1 \mathrm{~mm}$ cuvette was first illuminated with a $751 \mathrm{~nm} \mathrm{cw}$ laser $(1 \mathrm{~s}$, $500 \mathrm{~mW}$, Leading-Tech Laser) to produce the Pr-state. Flash photolysis was started with a $660 \mathrm{~nm}$ laser pulse from a tunable diode-pumped laser system ( $3 \mathrm{~ns}, 1.6 \mathrm{~mJ} \mathrm{~cm}^{-2}$, NT230, Ekspla). The spectrum after the flash was measured using a fiber optic halogen illuminator (7010, Cambridge Technology), a spectrograph 
(SpectraPro 150, Acton Research) and an intensified CCD camera (ICCD, InstaSpec V, Oriel) with time control by a delay generator (DG535, Stanford Research Systems). A long pass filter $>680 \mathrm{~nm}$ was used in the detection. The time window of illumination with probe light was synchronized to detection by using fast shutters (Vincent Associates) to obtain the same number of photons for each time point. After each flash, the sample was switched back to the Pr state by a $751 \mathrm{~nm}$ laser pulse (30 ms, $6.6 \mathrm{~mJ} \mathrm{~cm}^{-2}$ ), ensuring full conversion to Pr. Delay times were set in the region of $10 \mu \mathrm{s}-50 \mathrm{~ms}$ with a gate time of either $10 \mu \mathrm{s}$ or $1 \mathrm{~ms}$. Each time point was recorded 20 times at a $4 \mathrm{~Hz}$ repetition rate. Background spectra after applying only the $751 \mathrm{~nm}$ pulse were measured before and after the flash photolysis cycle and then averaged. To compensate for signal instabilities, the data was acquired with either increasing or decreasing delay times. In addition, all transmission spectra were normalized to the isosbestic point of the photoconversion as determined by steady-state UV-vis spectroscopy.

\subsection{Data analysis}

Analysis of the steady-state FTIR spectra was performed using Matlab version R2018b (The MathWorks, Inc). The set of difference spectra (Pfr minus Pr and vice versa) were averaged and offset corrected in the spectral range of 1981-1999 $\mathrm{cm}^{-1}$. Since the signal of the pAzF azide label was detected by difference spectroscopy, all the unchanged contributions were lost. To reconstruct the shape and position of the bands in $\mathrm{Pr}$ and Pfr, the signal at $2060-2180 \mathrm{~cm}^{-1}$ was fitted with the required number of Gaussian bands ${ }^{40,41,46,47}$ using the interactive peak fitter (ipf.m, version 13, available in https://terpcon nect.umd.edu/ toh/spectrum/InteractivePeakFitter.htm) with flat baseline correction.

The global analyses of time-resolved FTIR and flash photolysis data were performed with Glotaran 1.5.1. ${ }^{48}$ Ihalainen et $a l .{ }^{5}$ showed that the global analysis of time-resolved FTIR spectra of DrBphP $P_{\mathrm{PSM}}$ leads to SADS of two intermediate states, Lumi-R and Meta-R, and the photoproduct Pfr. Accordingly, a sequential model was applied here. The spectral region of water absorption (1610-1670 $\mathrm{cm}^{-1}$ ) in the FTIR spectra of pAzF mutants was ignored in order not to dominate the global analyses.

The time-resolved FTIR spectra of the wild type were scaled to those of Y472pAzF and F203pAzF using the negative $1562 \mathrm{~cm}^{-1}$ band maximum of the Pfr spectra. For Y176pAzF, this scaling procedure did not yield satisfactory results. Instead, spectra of the wild type were scaled to the negative $1712 \mathrm{~cm}^{-1}$ and positive $1685 \mathrm{~cm}^{-1}$ bands of Pfr in Y176pAzF. The steady-state spectra were scaled using the same factors.

\section{Results}

We successfully introduced the pAzF vibrational probe in three prime positions, Y472, F203, and Y176, in the phytochrome photosensory module $\operatorname{DrBphP}_{\mathrm{PSM}}$ (Fig. 1C). Important for our experimental settings, we find by UV-vis absorption spectroscopy that despite the effect of the azido group, the photo-stationary states ( $\mathrm{Pr}$ and $\mathrm{Pfr}$ ) of these samples are stable and can be interconverted repetitively (Fig. S1 and S2, ESI $\dagger$ ).

Y472pAzF reports particularly on the tongue region whereas Y176pAzF and F203pAzF provide information on the role of the hydrophobic pocket around the chromophore mainly formed by F203. Furthermore, Y176 contributes to the hydrophobic pocket, but in $\mathrm{Pfr}$ the $\mathrm{OH}$-group of tyrosine participates in a chromophore-stabilizing H-bond. ${ }^{7,11}$

On top of analyzing the pAzF signal of the three mutants, we also compare the photocycle of the pAzF mutants to the wild type, WT $D r B p h P_{\text {PSM }}$. FTIR spectroscopy captures the structural changes in both the protein moiety and the BV chromophore in the fingerprint region (1200-1765 $\mathrm{cm}^{-1}$ ), whereas UV-vis spectroscopy reveals the changes in the BV only. At steady-state, the difference spectra show structural changes from Pr to Pfr. The time-resolved experiments reveal the differences in structure of the intermediates to that of Pr. By means of global analysis of the time-resolved data, we compare the time constants as well as spectral properties of the intermediates during the photocycle. The direct comparison of time-resolved FTIR and UV-vis data reveals whether structural rearrangements in the protein proceed even after the chromophore has finalized its conversion.

We have chosen the monomeric form of the $\operatorname{DrBphP} \mathrm{PSM}^{44}$ to simplify the purification process by avoiding the separation of fully expressed homodimers from heterodimers with a truncated half because of the stop codon applied in the amber codon suppression technology (see Materials and methods). Monomeric DrBphP $P_{\mathrm{PSM}}$ undergoes the same photocycle as the dimer with similar FTIR as well as transient IR difference spectra and moreover provides clearer and more homogenous data than the dimer. ${ }^{5}$

As the phytochrome photoconversion between the Pr and Pfr state is such a complex process, additional sites would be desirable to label and investigate. Therefore, in addition to the positions mentioned above, we incorporated the probe at six other positions (Fig. S1, ESI $\dagger$ ). However, the probe at these positions either perturbed structural and photoswitching properties or did not lead to any or only minor changes in the steady-state FTIR difference signal in the azido region (Table S1, ESI $\dagger$ ). The UV-vis spectra (Fig. S2 and S3, ESI $\dagger$ ) and the steady-state FTIR difference spectra (Fig. S4, ESI $\dagger$ ) of the mutants are described in the Supplementary information including, where possible, difference spectra of the pAzF region (Fig. S5, ESI $\dagger$ ).

\subsection{Y472pAzF reports on the structural changes in the tongue}

Y472 is located in the so-called tongue region of the PHY domain (Fig. 1C). In Pr, the tongue has been suggested to form a $\beta$-sheet, but to convert to an $\alpha$-helix upon photoconversion to Pfr (Fig. 1A). ${ }^{11}$ As the structure of the tongue changes, the relative position and thus the microenvironment of the label at Y472 is also expected to change. The crystal structures of the photoactive states suggest that the side chain of Y472 is solvent exposed in Pr, but the $\alpha$-helical conformation of the tongue turns it towards the protein in Pfr (Fig. 1C). ${ }^{7,11}$ 
The microenvironment in Pr and Pfr is revealed by the pAzF vibrational frequency in the steady-state FTIR difference spectrum (Fig. 2A). Of note, the difference spectrum, i.e. Pfr minus Pr, allows a better resolution of the label signal than the absolute IR absorption spectrum (Fig. S6, ESI $\dagger$ ). In the difference spectrum, the negative signal represents the disappearance of absorption from the initial Pr state and the positive signal represents the induced additional absorption of the Pfr state. Therefore, an appearance of the azido signal in the FTIR difference spectrum at around $2100 \mathrm{~cm}^{-1}$ reports about a change in the local electronic environment of the label and a change of the $\mathrm{H}$-bonding strength between the label and its environment.

The pAzF signal in solution consists of an asymmetric azide stretch that is accompanied by two Fermi resonance bands resulting from anharmonic vibrational coupling between the asymmetric azide stretch and combination modes of the symmetric azide stretch and phenyl modes. ${ }^{47}$ To reveal the position of the contributing bands, the difference signal of Y472pAzF is fitted using a sufficient number of Gaussians (Fig. 2A). ${ }^{40,46,47}$ We assume that the frequency of the asymmetric azide stretch stays in the limits of $2110-2130 \mathrm{~cm}^{-1}$, according to the studies of Zhang et al. ${ }^{47}$ and Maj et al. ${ }^{40}$ Within these limits, an upshift in frequency indicates a larger $\mathrm{H}$-bond strength. ${ }^{38,40,41,47}$ Bands outside of these limits we identify as Fermi resonance bands. We consider it unlikely but cannot exclude the possibility that the main band and the Fermi resonance bands originate from two different protein conformations.

The steady-state FTIR difference spectrum of Y472pAzF reveals that the microenvironment of the probe at this position changes upon photoconversion. The azido vibration of Y472pAzF redshifts from $2125 \mathrm{~cm}^{-1}$ in Pr to $2114 \mathrm{~cm}^{-1}$ in Pfr with additional Fermi resonance bands (at $2141 \mathrm{~cm}^{-1}$ and $2105 \mathrm{~cm}^{-1}$ ) (Fig. 2A). The signals point to a strong H-bonding environment in Pr, and to a non- or weakly H-bonding environment in Pfr. ${ }^{38,40,41,47}$ Observations in the crystal structures support the information acquired from the FTIR spectrum, as an $\mathrm{H}_{2} \mathrm{O}$ of the solvent might be the H-bonding partner of Y472pAzF in $\mathrm{Pr}$, and its rotation from solvent exposure towards the protein moiety in Pfr then causes a loss of this strong H-bond network.

The small relative intensity of the azido peak compared to the fingerprint region is rationalized by recording the absolute absorption spectra of Y472pAzF in $\mathrm{D}_{2} \mathrm{O}$ (Fig. S6A, ESI $\dagger$ ). We find that the label signals in Pr and Pfr are almost completely overlapping and therefore compensate each other in intensity. This significant broadening of the bands might be caused by the dynamic nature of the refolding tongue in which the label in Y472pAzF is located in both Pr and Pfr states. ${ }^{49,50}$

By means of time-resolved step-scan FTIR spectroscopy, we demonstrate in which time scales the observed changes between Pr and Pfr arise at the different labeled locations. In the step-scan experiments, we selected the time window
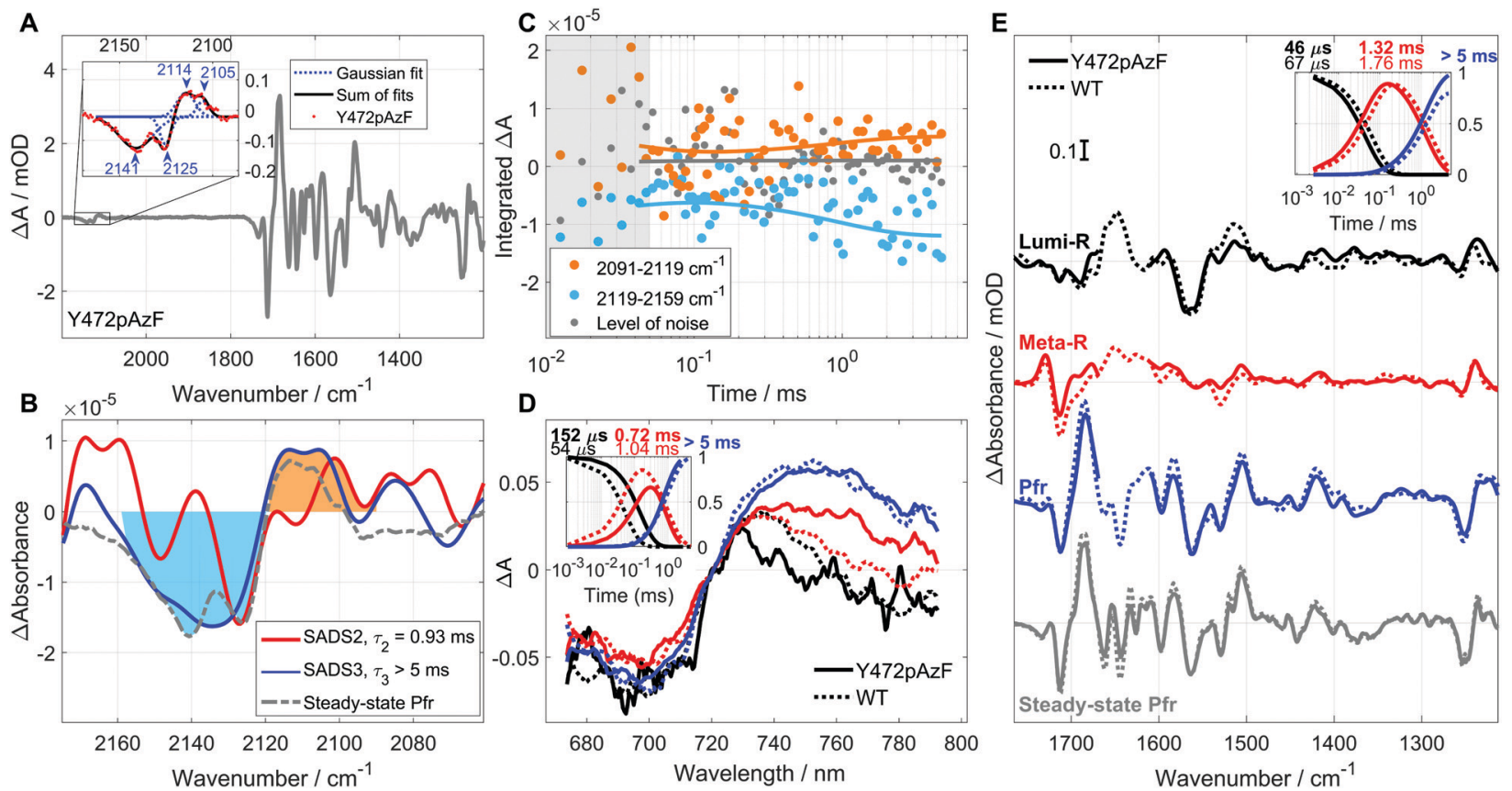

Fig. 2 Spectroscopic analysis of the Y472pAzF mutant of DrBphP्Ps. (A) The steady-state FTIR difference spectrum of the labeled sample. The signal of the label is fitted with four Gaussian bands (the inset), the maxima are indicated. (B) The global analysis of the time-resolved FTIR signal in the label region reveals three components. However, only two SADS are presented due to the large noise at early times $\left(\tau_{1}=35 \mu \mathrm{s}\right.$, see Fig. S7A, ESI $\left.\dagger\right)$. The steady-state spectrum scaling is the same as in (E). (C) Kinetics of the integrated spectral signal of the label (dots) with their fit (solid lines). The time constants are indicated in (B). The grey dots indicate the noise level of the experiment (Fig. S7A, ESI $\dagger$ ) and the grey background the time frame with high noise level. (D) SADS from the global analysis of the flash photolysis (vis to far-red detection) for Y472pAzF (bold) and WT (dotted) with their time constants. (E) Global analysis of the step-scan FTIR data in the fingerprint region for Y472pAzF vs. WT. The step-scan data at $1610-1670 \mathrm{~cm}^{-1}$ of the mutant is discarded due to dominating water absorption. The insets in (D) and (E) show the concentration fractions of the three different components in the analyses. 
from $2.5 \mu$ s to $4.7 \mathrm{~ms}$ to capture both the early and late steps of the photocycle. ${ }^{51}$

We evaluate separately the events in the label region (Fig. 2B and $\mathrm{C}$ ) and the photocycle of the pAzF-labeled mutants by global analysis ${ }^{52,53}$ of time-resolved FTIR spectroscopy (Fig. 2B and E) and flash photolysis in the visible region (Fig. 2D). To reveal the changes in the photocycle caused by the label or removal of the native residue, we compare the spectral fingerprint and associated kinetics to the unlabeled WT.

The global analysis in the region of $2060-2175 \mathrm{~cm}^{-1}$ shows that the label signal in Y472pAzF evolves in three components (SADS, species associates difference spectra), i.e., the site is responding to the activity in all three phases of the photocycle (Fig. 2B). Although the Lumi-R spectrum $(\tau=35 \mu \mathrm{s})$ is dominated by a rather large noise level (Fig. S7A, ESI $\dagger$ ), the early changes are clearly revealed by integrating the data of the label signal from 2091 to $2119 \mathrm{~cm}^{-1}$ and 2119 to $2159 \mathrm{~cm}^{-1}$; these regions are indicated in Fig. $2 \mathrm{~B}$ in orange and blue, respectively. The integrated data is plotted in Fig. $2 \mathrm{C}$ in comparison to the noise level, as a result we excluded the earliest data points from the analysis. The change in absorbance of the label appears already at $40 \mu \mathrm{s}$. The integrated amplitude of both positive and negative signals at $40 \mu \mathrm{s}$ is only about half of the integrated signal intensity in Pfr, but clearly above the noise level. This finding indicates that the label environment undergoes structural changes already in the early microsecond time scale.

In the Meta-R spectrum, four bands (two negative and two positive) similar to the bands in the final photoproduct are observed, but with different amplitude ratios (Fig. 2B). Moreover, the integrated difference signals continue to grow with the time constant of $0.93 \mathrm{~ms}$ (Fig. 2C). This analysis reveals that the evolution of the structural changes in the tongue towards the final Pfr environment is complex, because the label experiences environments different from Pr and Pfr during the conversion process. The final SADS assigned to the Pfr state matches the steady-state Pfr spectrum (Fig. 2B), which means that the label settles to its final Pfr environment within the time frame of the experiment $(4.7 \mathrm{~ms})$. Accordingly, the final restructuring of the tongue occurs during the final step of the photocycle from Meta-R to Pfr, in agreement with previous analysis of the amide I and II signals in WT. ${ }^{5}$

The photoconversion of the labeled phytochromes is followed by flash photolysis, which shows the changes in the chromophore absorption (Fig. 2D). For comparison, the recorded stepscan FTIR spectra in the fingerprint region (below $1765 \mathrm{~cm}^{-1}$ ) reveal the changes in chromophore and protein vibrations during the photocycle (Fig. 2E). In the global analysis, both data (flash photolysis and step-scan FTIR) are fitted with three components, leading to spectra of Lumi-R, Meta-R, and Pfr states, respectively. The three SADS of the step-scan experiment are confirmed by extracting raw data averaged according to the species concentrations in time (Fig. S8, ESI $\dagger$ ).

The changes in the chromophore and the protein moiety proceed with similar kinetics as the spectral signatures of the label environment of Y472pAzF. The time constant of the label Lumi-R (35 $\mu \mathrm{s}$ ) is very close to that of the fingerprint region (Fig. 2E).
The time constant of the label for Meta-R lies in between the time constants observed in the flash photolysis and in the FTIR fingerprint region (numeric values indicated in Fig. 2B, D and E). We note that slight differences are expected as the chromophore, protein and label report different information on the photoconversion process. $^{45}$

In comparison with the WT, the photocycle of $\mathrm{Y} 472 \mathrm{pAzF}$ proceeds highly similarly with regards to spectral features as well as kinetics (Fig. 2E). The first intermediate Lumi-R is observed with matching spectral characteristics and a time constant comparable to the WT. The Meta-R state decays slightly faster than in WT and shows characteristic features, e.g., the positive peak at $1730 \mathrm{~cm}^{-1}$. Finally, the labeled phytochrome reaches, like the WT, a Pfr spectrum that strongly resembles the steady-state spectrum, considering that the spectral resolution of the time-resolved experiments was $8 \mathrm{~cm}^{-1}$ as compared to $2 \mathrm{~cm}^{-1}$ in the steady-state spectra.

The global analysis of the flash photolysis reveals slight differences in kinetics between the mutant and the WT (Fig. 2D). In Y472pAzF, the Lumi-R state decays slower than in WT and on the other hand, the Meta-R decays a bit earlier, leading to a lower population of Meta-R in the mutant. The difference spectrum at $5 \mathrm{~ms}$ has the same features as the steady-state difference spectrum, meaning that the photocycle of the chromophore has been finalized by this time. In fact, in all three mutants the chromophore finishes the photocycle within $5 \mathrm{~ms}$, as their latest flash photolysis spectra match the steady-state absorption difference spectra (Fig. S9 and S10, ESI $\dagger$ ).

\subsection{Y176pAzF demonstrates major hydrogen bonding change in the late phase of the photoconversion}

Y176 is a buried residue, located in the chromophore binding pocket near the BV D-ring in the DrBphP $_{\mathrm{PSM}}$ (Fig. 1C). In Pr, it is part of the hydrophobic pocket stabilizing the D-ring position. ${ }^{6,11}$ In the transition to Pfr, it moves together with F203 to a new location, where it stabilizes the Pfr state. ${ }^{7,45,54}$

The steady-state FTIR difference spectrum of Y176pAzF shows that the azido vibrations blueshift from $2112 \mathrm{~cm}^{-1}$ in Pr to $2125 \mathrm{~cm}^{-1}$ in Pfr with an additional Fermi resonance band at $2136 \mathrm{~cm}^{-1}$ (Fig. 3A and Fig. S6B, ESI $\dagger$ ). Further, a slight negative feature can be discerned at $2071 \mathrm{~cm}^{-1}$ in $\mathrm{Pr}$, arising due to different peak widths possibly because of Fermi resonances. The intensity of the difference signal is larger in the case of Y176pAzF than for Y472pAzF, which is caused by a larger shift of the peak maximum between Pr and Pfr (Fig. S6B, ESI $\dagger$ ).

Based on the observed frequencies $\left(2112 \mathrm{~cm}^{-1}\right.$ in $\operatorname{Pr}, 2125 \mathrm{~cm}^{-1}$ in Pfr), the azido group in Y176pAzF has mainly a non- or weakly $\mathrm{H}$-bonding environment in the Pr state but a strong H-bond in the Pfr state. ${ }^{19,39,47}$ The interpretation of the environments is supported by the same frequencies as in the case of Y472pAzF (Fig. 2A). However, as the side chain of Y176 is not solvent-exposed, the strong H-bonding partner of the label (or the native tyrosine) needs to locate inside the protein. Therefore, candidates for the H-bonding partner(s) are the chromophore, another residue, or intramolecular water, of which 
A

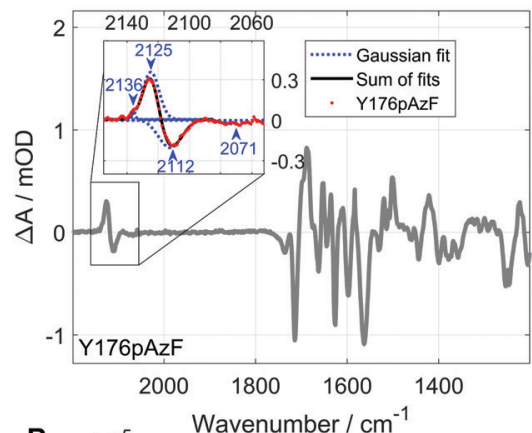

B

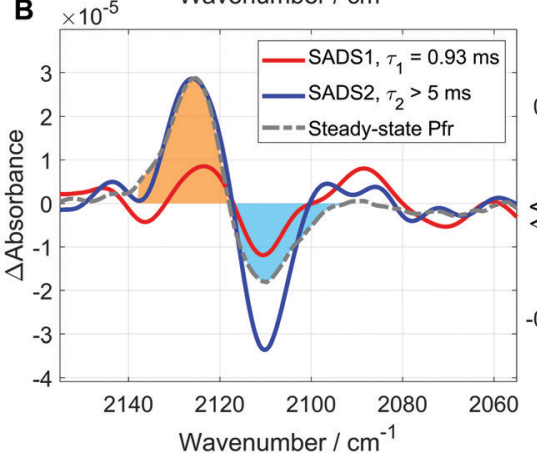

C

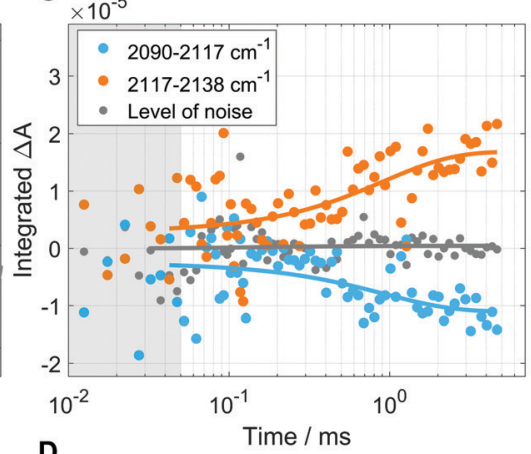

D

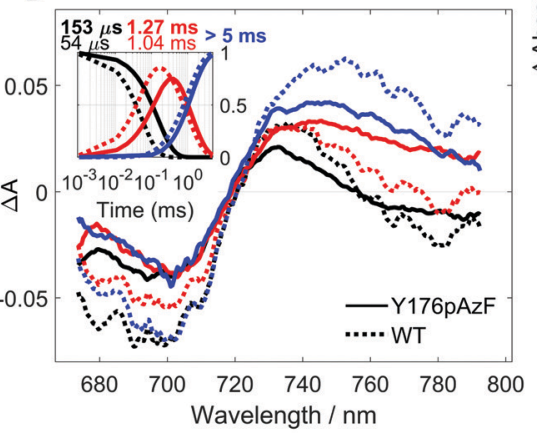

$\mathrm{E}$

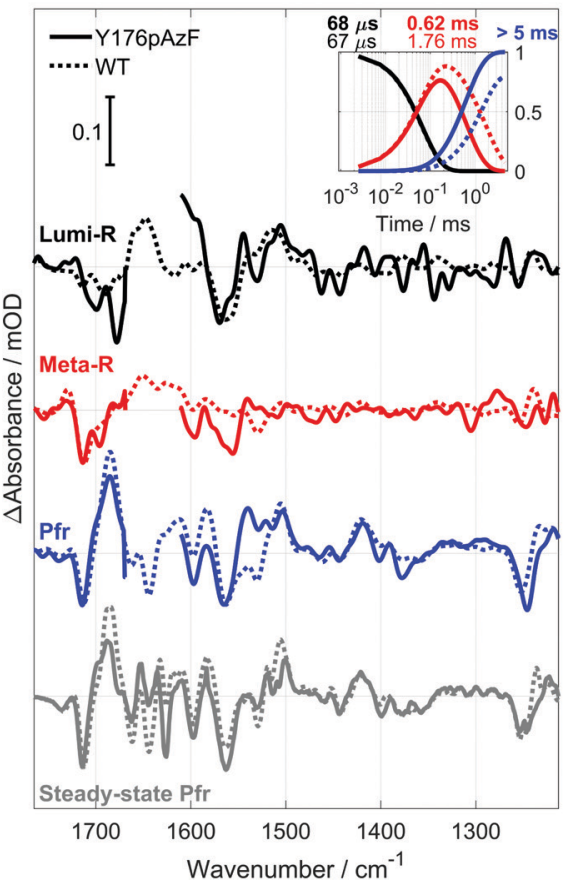

Fig. 3 Spectroscopic analysis of the Y176pAzF mutant of DrBphP PSM. (A) A prominent label signal in the Y176pAzF steady-state FTIR difference spectrum is fitted with four Gaussian bands (the inset), the maxima positions are labeled. (B) The global analysis of the pAzF signal reveals two SADS. The scaling of the steady-state spectrum is the same as in (E). (C) Kinetics of the integrated spectral signal of the label (dots) and their multiexponential fit (line) with the time constants given in (B). The grey dots indicate the noise level (Fig. S7B, ESI $\dagger$ ), and the grey background the time frame with high noise level. (D) The flash photolysis SADS after global analysis with the resulting time constants, Y176pAzF (bold) and WT (dotted). (E) Global analysis in the FTIR fingerprint region of Y176pAzF vs. WT. The data at $1610-1670 \mathrm{~cm}^{-1}$ is discarded due to dominating water absorption. The insets in (D) and (E) show the concentration fractions of the three different components.

the latter would most probably offer the strongest H-bonding in agreement with the azide frequency.

In the time-resolved data, the global analysis of the label region reveals only one intermediate, Meta-R, between Pr and Pfr of the label (Fig. 3B). The lifetime of the Meta-R state is $0.93 \mathrm{~ms}$. We interpret this finding as a change in the microenvironment that takes place only in the transition from the Pr to the Meta-R state, and then further to the Pfr state. The SADS signals have similar lineshapes in the transition from $\operatorname{Pr}$ to Meta-R and from Meta-R to Pfr, which suggests that the events in the microenvironment of Y176pAzF are a step-wise process.

The shift of the label frequency starts to appear with a larger amplitude only at around $300 \mu \mathrm{s}$, long after the decay of the Lumi-R state (Fig. 3C). Therefore, the formation of the new $\mathrm{H}$-bond between the residue and the environment is locked in the Meta- $\mathrm{R} \rightarrow$ Pfr transition. We note that the label signal does not reach its final state during the experimental time window of $4.7 \mathrm{~ms}$. In particular, the ratio of the positive and negative band of the label does not match between the step-scan and steady-state Pfr difference spectra, but shows additional positive features in the step-scan spectrum below $2100 \mathrm{~cm}^{-1}$ (Fig. 3B). It is thus likely that once an amino acid responsible for stabilizing the Pfr state is modified, the final equilibration takes longer than the time window of our experiment. In contrast, the results from flash photolysis indicate that the Pr to Pfr conversion of BV is already completed within $5 \mathrm{~ms}$ (Fig. S9, ESI $\dagger$ ), which indicates that a late relaxation process takes place close to the label that does not affect the BV.

The global analyses of the Y176pAzF fingerprint region from the time-resolved FTIR experiments and of the visible region from flash photolysis reveal that the photocycle of the mutant proceeds via two intermediates, Lumi-R and Meta-R (Fig. 3D and E). As observed by time-resolved FTIR, the Lumi-R state in Y176pAzF and WT and decays with identical time constants (Fig. 3E). In the visible, the Lumi-R and Meta-R intermediates of the Y176pAzF decay slower than in the WT (Fig. 3D). Accordingly, the label appears to slow down the Lumi-R decay from the perspective of the chromophore, in a similar way as in Y472pAzF. In contrast, the time-resolved FTIR shows an almost three times shorter lifetime for Meta-R of the mutant than that of the WT (Fig. 3E).

The spectral character along the photocycle clearly deviates between Y176pAzF and WT (Fig. 3D and E). The flash photolysis and steady-state UV-vis spectra of Y176pAzF reveal that the mutant converts between Pr and Pfr, but as the spectra differ from those of the WT, the label alters the environment of BV in the binding pocket (Fig. S3, ESI $\dagger$ ). Focusing on the FTIR features, notable differences are detected already in the Lumi-R intermediate. The negative $1714 \mathrm{~cm}^{-1}$ band in WT, assigned to the BV D-ring carbonyl vibration, ${ }^{14}$ is visible only as a shoulder in the Lumi-R spectrum of the mutant. A negative/positive pattern at $1699(-) / 1689(+) \mathrm{cm}^{-1}$, which indicates a shift of an absorption 
band, locates in the mutant at higher frequencies than in the WT $\left(1689(-) / 1678(+) \mathrm{cm}^{-1}\right)$.

The Y176pAzF Lumi-R spectrum shows much larger negative bands at 1699 and $1678 \mathrm{~cm}^{-1}$ than in the case of the WT, which shows only a small negative signal at $1672 \mathrm{~cm}^{-1}$. This feature indicates stronger changes in the water and/or protein signals compared to the WT. The negative sign implies that labeling of the Y176 changes not only the Pfr, but also the Pr structure of the protein. These prominent signals might be characterized more closely by ultrafast experiments on the Lumi-R state of Y176pAzF, which are to be performed in the future. Of note, the prominent amide II contribution of Lumi-R at $1564(-) \mathrm{cm}^{-1}$ in the WT is preserved in the mutant, which reflects comparable secondary structural changes in this phase of the photocycle.

In the Meta-R spectrum, the mutant shows the characteristic but smaller positive $1730 \mathrm{~cm}^{-1}$ signal and the negative signal at $1712 \mathrm{~cm}^{-1}$, which form the difference band assigned to the $\mathrm{BV}$ D-ring carbonyl group that lost the H-bond of the Pr state and has not yet formed the interactions present in the Pfr state. ${ }^{5}$ A notable change to the WT spectrum is the presence of an amide II mode indicating secondary structural changes in the $1550 \mathrm{~cm}^{-1}$ region, as in the case of the WT, this region is silent in the Meta-R state. ${ }^{5}$ At $4.7 \mathrm{~ms}$, the Pfr spectrum of Y176pAzF does not yet correspond to that of the steady-state Pfr as the ratio of the D-ring carbonyl signals $\left(1712(-) / 1685(+) \mathrm{cm}^{-1}\right)$ notably changes and the $1540(+) \mathrm{cm}^{-1}$ band at $4.7 \mathrm{~ms}$ is missing in the steady-state Pfr. These changes underline that the protein still undergoes changes after the experimental time window, which is in agreement with the label region of the Pfr spectra (Fig. 3B). In contrast, flash photolysis demonstrates that the chromophore completes the photocycle within $5 \mathrm{~ms}$, as the last time-resolved spectrum corresponds to the steady-state UV-vis spectrum (Fig. S9B, ESI $\dagger$ ). Hence, some late structural changes or protein relaxation processes take place independently from the chromophore.

The labeling of Y176 causes a loss of a vibrational band at $1236(-) \mathrm{cm}^{-1}$ in the Pfr difference spectrum when compared to WT (Fig. 3E). This loss is clearly observed in the double difference spectrum of Y176pAzF and WT (Fig. S11, ESI $\dagger$ ). The $1236 \mathrm{~cm}^{-1}$ signal is present even after ${ }^{13} \mathrm{C}^{15} \mathrm{~N}$ global labeling of the protein moiety, ${ }^{5}$ which points to $\mathrm{BV}$ as the major origin. To verify that the signal loss is an effect of replacing the tyrosine, we included the $\mathrm{Y} 176 \mathrm{~F}$ mutant in the analysis. For Y176pAzF and Y176F, bands were observed in the double difference of Pfr at 1236 and $1234 \mathrm{~cm}^{-1}$, respectively (Fig. S11, ESI $\dagger$ ). These findings imply that the tyrosine residue plays an important role in stabilizing the BV conformation in Pfr. For both mutants, the double difference spectrum shows a positive signal at $1506 \mathrm{~cm}^{-1}$ and negative signals at $1529 \mathrm{~cm}^{-1}$ (Y176pAzF) and $1526 \mathrm{~cm}^{-1}$ (Y176F) (Fig. S11, ESI $\dagger$ ). These signals might be attributed to the missing tyrosine residue as they are near the frequencies reported for $\nu \mathrm{CC}$ of the tyrosine phenyl ring. ${ }^{55,56}$

A closer look at the time-resolved data reveals that the $1236 \mathrm{~cm}^{-1}$ signal missing in Y176pAzF is clearly visible in only the Meta-R and Pfr states of the WT, but not in Lumi-R (Fig. 3E).
This finding is in line with the time scales of conformational realignment determined from the label signal of Y176pAzF (Fig. 3B and C). Like the label, the native Y176 becomes active only in the Meta-R intermediate state.

\subsection{The early stage action of F203pAzF is followed by accelerated Pfr formation, diminishing Meta-R}

F203 is located close to the D-ring of the BV and is part of the hydrophobic pocket that stabilizes the position of the D-ring in the Pr state (Fig. 1C). According to the crystal structures obtained from $\operatorname{DrBphP}_{\mathrm{PSM}}{ }^{7,11}$ the residue is however not fully buried inside the chromophore binding domain. The phenylalanine side chain is exposed to the structurally flexible tongue region in Pr, but expected to turn away from the tongue in Pfr. ${ }^{7}$

In F203pAzF, the azide vibrational frequency redshifts from $2122 \mathrm{~cm}^{-1}$ in $\operatorname{Pr}$ to $2106 \mathrm{~cm}^{-1}$ with an additional weaker contribution at $2153 \mathrm{~cm}^{-1}$ in Pfr (Fig. 4A). The frequencies were confirmed with lower resolution absorbance spectra that showed similar frequencies (Fig. S6C, ESI $\dagger$ ). As neither of the two peaks in Pfr fit to the reported pAzF frequencies, ${ }^{40}$ it is assumed that the original Pfr signal has split symmetrically into the two observed signals because of a resonance with combinations and overtones of modes with lower frequency. For example, the $\nu \mathrm{CC}$ vibration of AzF has a frequency of $1036 \mathrm{~cm}^{-1}$ (according to quantum chemical calculations), so that the overtone would be slightly below $2072 \mathrm{~cm}^{-1}$ and could couple with the azido vibration in Pfr, if close to this frequency. In a simplified approach to reconstruct the original azido frequency, the midpoint of the observed frequencies of 2106 and $2153 \mathrm{~cm}^{-1}$ would be at $2129 \mathrm{~cm}^{-1}$. Accordingly, such a reconstructed frequency might point to a strongly $\mathrm{H}$-bonding environment in Pfr, whereas the detected signal at $2122 \mathrm{~cm}^{-1}$ in Pr results from a weaker/non H-bonding environment. ${ }^{40}$

In the global analysis of the time-resolved data, the label signal of F203pAzF needs three components (Fig. 4B). The first intermediate decays with a time constant of $35 \mu \mathrm{s}$ and is interpreted as a Lumi-R state spectrum as discussed below in the context of the FTIR fingerprint region and the flash photolysis. The second intermediate has a time constant of $1.2 \mathrm{~ms}$. In addition, the global analysis reveals that the label signal goes through an intermediate (SADS3) that has a different lineshape than the final signal in Pfr, which indicates that the label undergoes multiple processes during the photocycle rather than a single event as for Y176pAzF (Fig. 3B).

The integrated signals are followed based on the limits of the Pfr label signal (Fig. 4C). At $40 \mu \mathrm{s}$, the label frequency has already shifted, observed as increased integral absorption difference of both positive and negative signals. The amplitude of the signals increases up to $1 \mathrm{~ms}$ and then stays constant, which implies that the label senses a conformational change. However, after $4.7 \mathrm{~ms}$, the amplitude at $2106 \mathrm{~cm}^{-1}$ increases further and the prominent signal at $2153 \mathrm{~cm}^{-1}$ should fully decay to match the steady-state difference spectrum. Accordingly, the label has not reached its final microenvironment within the time frame of the experiment. 
A
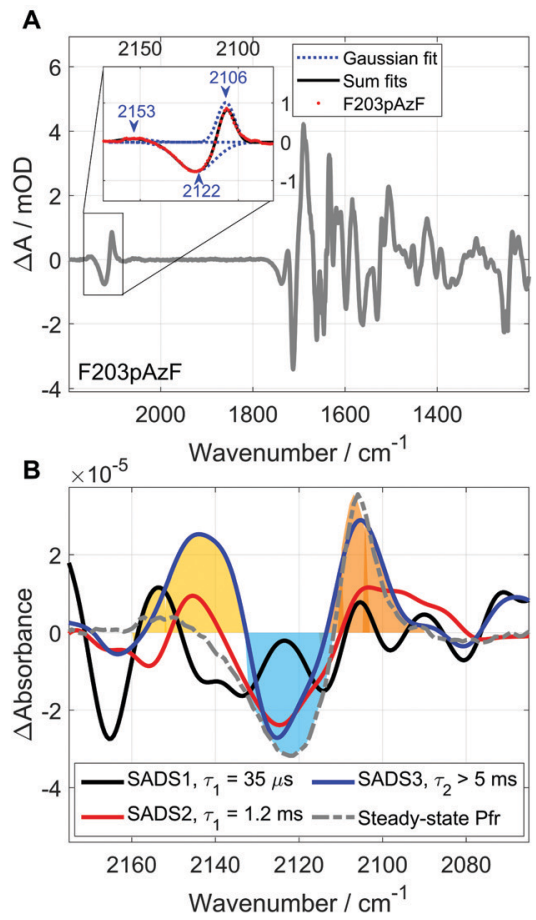

C $\times 10^{-5}$
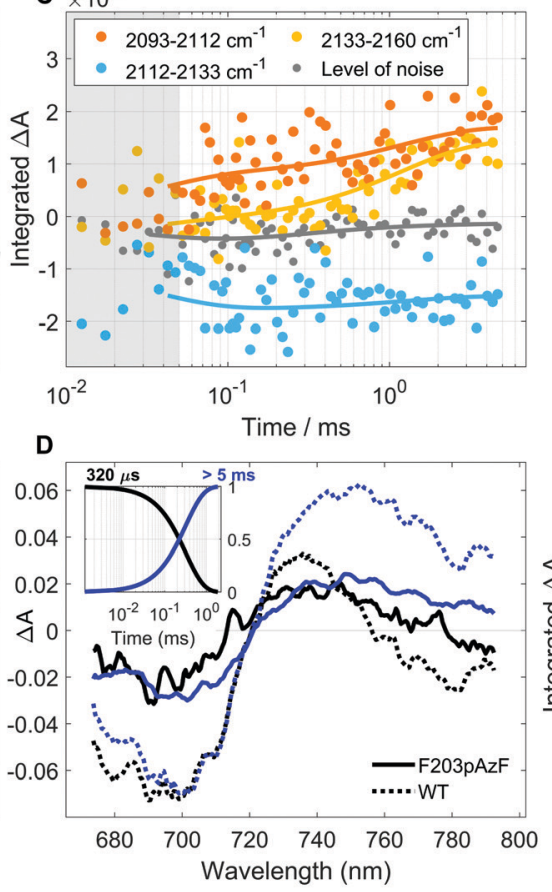

E

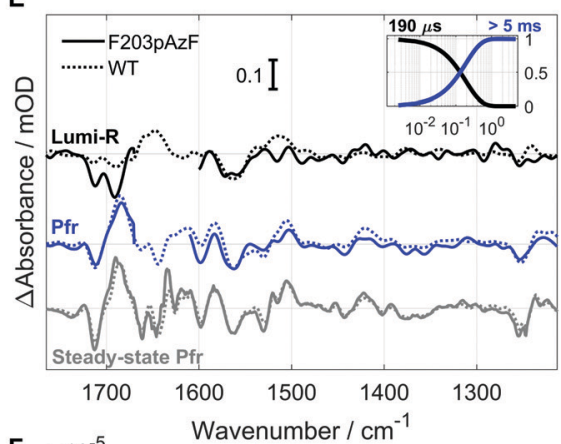

$\mathbf{F} \times 10^{-5}$

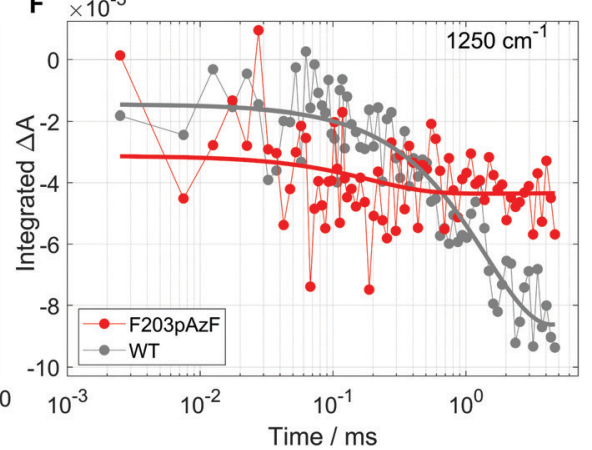

Fig. 4 Spectroscopic analysis of the F203pAzF mutant of DrBphPPS. (A) The steady-state FTIR difference spectrum of F203pAzF shows a prominent label signal, which is fitted with three Gaussian bands and the positions of the maxima are labeled (the inset). (B) The global analysis of the label region reveals three SADS. The steady-state spectrum is scaled as in (E). (C) Kinetics of the integrated spectral signal of the label (dots) and the multiexponential fit (lines) with the time constants given in (B). The grey dots represent the noise level (Fig. S7C, ESI $\dagger$ ) and the grey background the time frame with high noise level. (D) SADS from the global analysis of the flash photolysis for F203pAzF (bold) and WT (dotted). (E) Global analysis of step-scan FTIR data of the mutant vs. WT. The data at $1610-1670 \mathrm{~cm}^{-1}$ is discarded due to dominating water absorption. The insets in (D) and (E) show the concentration fractions of the three different components. (F) The kinetics of the $1250 \mathrm{~cm}^{-1}$ signal from BV (integrated at $1245-1255 \mathrm{~cm}^{-1}$ ) of F203pAzF vs. WT.

The global analysis of the label revealed three SADS with very similar time constants to the WT photocycle (Fig. 4B). However, the global analysis of the F203pAzF time-resolved data of the fingerprint region reveals that the mutant skips the Meta-R intermediate, because all data can be fitted by only two components instead of three. The FTIR fingerprint region and flash photolysis reveal very similar time constants for the first intermediate with $190 \mu$ s and $320 \mu$ s, respectively (Fig. 4D and E). In the Supplementary information an alternative fit with three components is demonstrated, of which the second intermediate lacks the characteristics of Meta-R but resembles a superposition of Lumi-R and Pfr spectra (Fig. S12, ESI $†$ ). This interpretation is underlined by the kinetics of the $1250 \mathrm{~cm}^{-1}$ signal, which originates mainly from the BV, according to ${ }^{13} \mathrm{C}^{15} \mathrm{~N}$ labeling of the protein moiety. ${ }^{5}$ In F203pAzF, the signal is finalized early, whereas in WT the rise starts only after hundreds of microseconds and is finalized within a few milliseconds (Fig. 4F).

Labeling of F203 leads to changes in the Lumi-R spectrum at around $1695 \mathrm{~cm}^{-1}$ and $1670 \mathrm{~cm}^{-1}$ similar to those introduced by Y176 labeling (Fig. 4E). In F203pAzF, the negative band is located at $1691 \mathrm{~cm}^{-1}$ and the positive at $1672 \mathrm{~cm}^{-1}$. The amplitude is much stronger than in the case of WT. The large negative contribution in this region even further decreases the D-ring carbonyl signal at $1712(-) \mathrm{cm}^{-1}$. The most plausible explanation for the $1691 \mathrm{~cm}^{-1}$ negative signal is an
$\mathrm{H}_{2} \mathrm{O}$ molecule, either in the interior of the protein or at the protein surface, which displaces due to switching to the Lumi-R-state.

In the Pfr state, the F203pAzF mutant shows a similar steady-state FTIR difference spectrum to WT. However, the step-scan Pfr spectrum of F203pAzF differs slightly from the steady-state spectra as the A- and D-ring signals between $1680-1730 \mathrm{~cm}^{-1}$ reveal different amplitude ratios. In contrast, the visible absorption spectrum from flash photolysis after $5 \mathrm{~ms}$ resembles the final Pfr spectrum (Fig. S9, ESI $\dagger$ ). Therefore, we interpret that the interactions chromophore are finalized to a Pfr orientation but additional changes take place after $5 \mathrm{~ms}$ in the label environment, similarly to the Y176pAzF mutant.

\subsection{The effects of the azido group on the photocycle}

The pAzF label at each studied site provides us local information on the signal propagation during the photocycle. However, the replacement of the native residues with the labeled amino acid with a strong dipole in the azido group can affect the photocycle. This effect then enlightens the role of the native residues in the photocycle. We focus on the kinetic traces at $1712(-) \mathrm{cm}^{-1}$ and $1730(+) \mathrm{cm}^{-1}$, which report on the BV D-ring carbonyl signals (Fig. $5 \mathrm{~A}-\mathrm{C}$ ), as well as on the traces at $1508(+) \mathrm{cm}^{-1}$ and $1564(-) \mathrm{cm}^{-1}$ to follow the secondary structural changes in the amide II region (Fig. 5D-F). The negative $1712 \mathrm{~cm}^{-1}$ signal evolves as the BV D-ring carbonyl moves from its H-bonding 

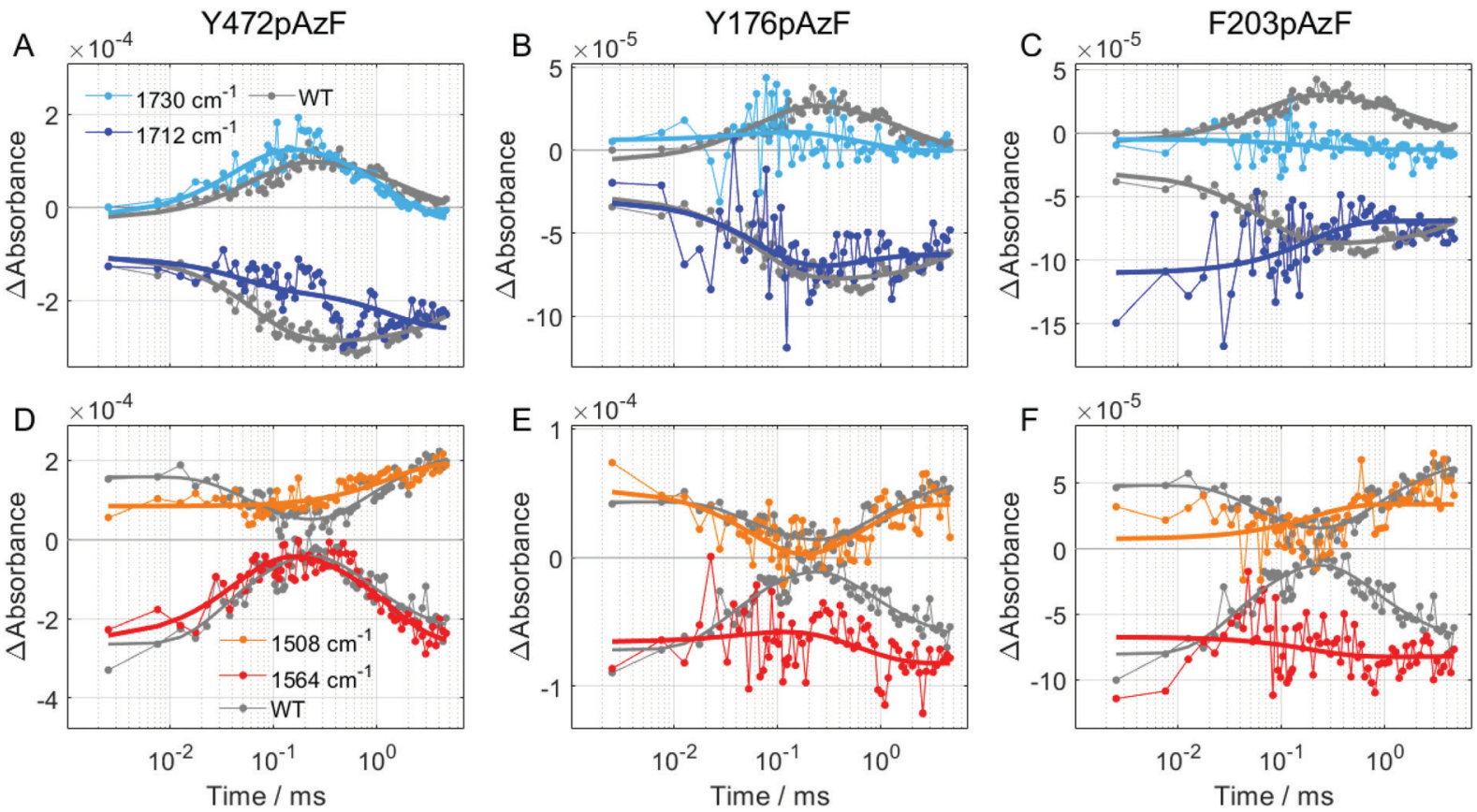

Fig. 5 Comparison of the pAzF mutants and WT signals in the BV and amide II regions. (A-C) The kinetic traces of the $1730 \mathrm{~cm}^{-1}$ (light blue) and $1712 \mathrm{~cm}^{-1}$ (blue) signals visualize the changes in the environment of the BV D-ring carbonyl in comparison to the WT (grey). (D-F) The kinetic traces of the amide II signals at $1508 \mathrm{~cm}^{-1}$ (orange) and $1564 \mathrm{~cm}^{-1}$ (red) of the mutants are compared those of the WT (grey). Fits are shown with solid lines. The time constants are given in Fig. 2-4E.

network in the Pr state via Lumi-R and Meta-R to that of Pfr. In Meta-R, the signal at $1730 \mathrm{~cm}^{-1}$ rises as characteristics of a D-ring carbonyl without H-bonding partners. ${ }^{5}$

In Y472pAzF, the D-ring carbonyl traces resemble those of the WT (Fig. 5A). The $1730 \mathrm{~cm}^{-1}$ signal rises slightly earlier than in WT in agreement with a faster Lumi-R decay in stepscan and flash photolysis. The $1712 \mathrm{~cm}^{-1}$ signal shows a uniform decrease in the final amplitude of the WT without the decrease and rise related to the Meta- $\mathrm{R}$ state. This difference is possibly due to the weaker signal at $1712 \mathrm{~cm}^{-1}$ in the Meta-R spectrum. Also the amide II traces are very similar in Y472pAzF and WT (Fig. 5D). The traces reveal that secondary structural changes take place already in the early phase of the photocycle but are finalized during the Meta-R to Pfr transition. This feature was also reported by the label at Y472pAzF (Fig. 2B). The photocycle of Y472pAzF follows very well the characteristics of the WT (Fig. 2D and E), which implies that either the hydroxyl group of the tyrosine is not crucial for the photocycle, or that the azido group of the label acts in a similar way to the tyrosine in its native position.

The traces of Y176pAzF follow the characteristics of the WT traces (Fig. 5B and E). The most notable difference is the lower amplitude of the $1730 \mathrm{~cm}^{-1}$ signal, which is explained by the lower Meta-R concentration in the mutant (Fig. 3D and E). Furthermore, the $1564 \mathrm{~cm}^{-1}$ trace does not decay towards Meta-R in Y176pAzF as in WT. A closer look at the amide II range of the Meta-R spectrum (Fig. 3E) reveals that the Meta-R intermediate in $\mathrm{Y} 176 \mathrm{pAzF}$ already has characteristics of Pfr. Although Y176pAzF mostly follows the photocycle of WT, the data demonstrate that the native tyrosine stabilizes the Meta-R state of the protein because the insertion of the label suppresses the Meta-R features.

Contrary to the other mutants, the replacement of F203 with pAzF causes more notable changes in the photocycle. The $1730 \mathrm{~cm}^{-1}$ trace does not reveal any significant positive contribution in F203pAzF (Fig. 5C) in agreement with the fact that the global analysis does not show a recognizable Meta-R intermediate (Fig. 4E and Fig. S12, ESI $\dagger$ ). The $1564 \mathrm{~cm}^{-1}$ trace behaves similarly for F203pAzF as for Y176pAzF, in which the Meta-R is suppressed (Fig. 5E and F). However, the biggest difference compared to the WT is observed in the $1712 \mathrm{~cm}^{-1}$ trace that in F203pAzF already has a high intensity during the first microseconds of the photocycle and then decays towards Pfr. This finding indicates that by addition of the azide moiety, the Lumi-R conformation of the chromophore is different than in WT. Furthermore, the F203 residue seems to act like a gatekeeper that forces the protein to the Meta-R intermediate. The large dipole moment of the azide group might guide the residue to a different position than in WT, which prevents F203 from fulfilling its role in the photocycle. We would like to stress that F203pAzF finally forms a photostable Pfr state, which, however, is slightly different from the WT Pfr both in terms of the steady-state UV-vis and FTIR difference spectra (Fig. S2 and S4, ESI $\dagger$ ).

\section{Discussion}

\subsection{Insights from the labels into the mechanism of phytochromes}

Phytochromes undergo a complex photoconversion from Pr to Pfr state upon red light excitation. In the beginning of the 
photocycle, the chromophore binding pocket is the hotspot of action as the whole process is triggered by the isomerization of the BV D-ring. Here, photoinduced changes in three different positions, Y472 in the tongue and Y176 and F203 near the isomerizing BV D-ring, were followed by replacing the residues with the sensitive IR-probe pAzF. We were able to record the changes in the local environment of these labels in a timeresolved manner and thus deduce the time span of action of each residue during the photocycle. The response at the three positions to the photoconversion of $\mathrm{BV}$ is summarized in Fig. 6A, in which the coloring of the circles highlights changes during the lifetime of the two intermediates Lumi-R and Meta-R, the photoproduct Pfr and events taking place after the experimental time window of $5 \mathrm{~ms}$.

We note that according to flash photolysis, the three mutants finalize the photocycle within $5 \mathrm{~ms}$, detected as a vis-to-far-red signal from the chromophore. Nevertheless, both of the labels in the chromophore binding pocket report on changes taking place over tens of milliseconds (Fig. 3B and 4B). Many other (bacterio)phytochromes such as Cph1 and Agp $1^{57,58}$ show a more complex photocycle with a split Meta-R state (Meta-Ra and Meta-Rc), of which the lifetime of the Meta-Rc state is even hundreds of milliseconds. The associated proton release-uptake event has not been reported for DrBphP and therefore, the lifetime of the photocycle is only in the order of few to tens of milliseconds. ${ }^{4}$ Still, large-scale changes of the $\operatorname{DrBphP}_{\mathrm{PSM}}$ protein occur in the time-range of tens of milliseconds as detected by X-ray scattering. ${ }^{4}$ The late signals from the labels underline the multi-dimensional nature of the photocycle: the photoconversion processes on different levels (tiers) of the protein are only partially coupled. ${ }^{459}$ Thus, the Pr or Pfr state can be reached in the chromophore even though in other sections the protein is still in the non-equilibrium state.

Y472pAzF allowed us to follow the reorganization of the tongue. We show that the tongue is in action already in the early phase (Fig. 2) and that the response continues until the end of the photocycle. Importantly, the changes in the tongue end at the same time as the chromophore finishes its photocycle underlining the tight connection of the tongue to the chromophore. The photocycle of the Y472pAzF mutant appears to be very similar to that of the WT (Fig. 2D, E, Fig. S4 and S2, ESI $\dagger$ ). Moreover, the label signal (Fig. 6B) propagates in a similar manner as the secondary structural changes during the photocycle (Fig. 5D), which further underlines the labels effectiveness in reporting the events in the refolding tongue.

The label Y176pAzF revealed that the residue forms a strong $\mathrm{H}$-bond in the Pfr state (Fig. 3A and B). The observed frequency for Y176pAzF is the same as for the solvent-exposed Y472pAzF. However, Y176 is buried and not in direct contact with solvent waters. Instead, upon photoconversion it turns away from the hydrophobic pocket in Pr towards the BV C-ring propionate (Fig. 1C). As the propionate cannot form an $\mathrm{H}$-bond with $\mathrm{pAzF}$ and based on the observed frequencies, we propose that the $\mathrm{H}$-bonding partner is an intramolecular water positioned between Y176 and the C-ring propionate. This, however, remains to be validated in future studies. The residue becomes active only in the late phase of the photocycle (Fig. 3B and C) as reflected in both the label and the tyrosine signals. Therefore, Y176 most probably has a preparative role in the photocycle within the chromophore binding pocket, and the H-bond remains as a part of the network stabilizing the Pfr conformation, possibly via the propionate.
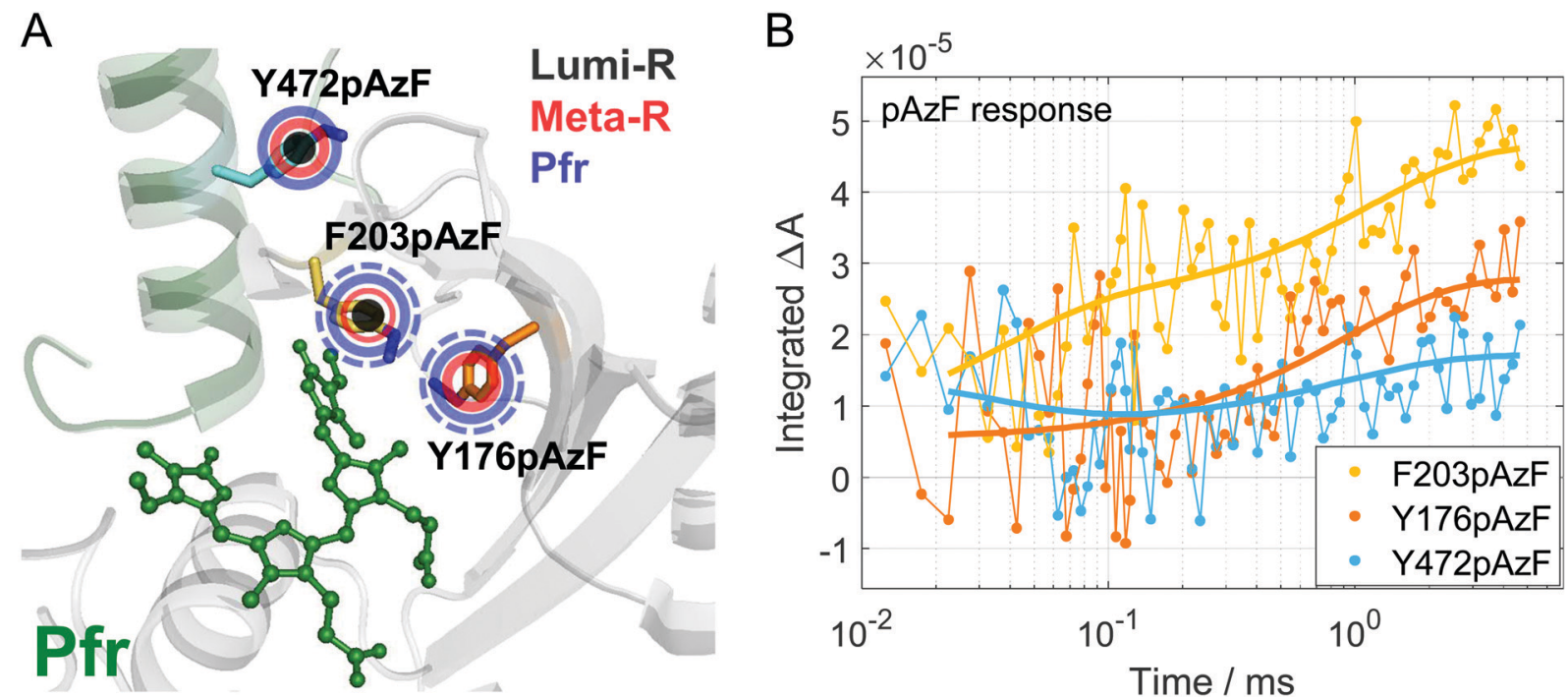

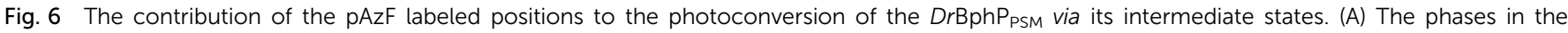

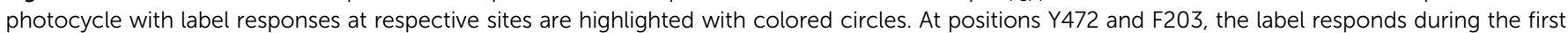

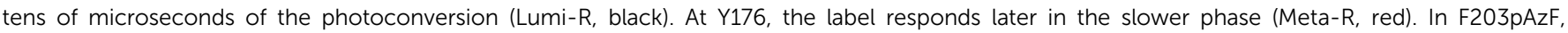

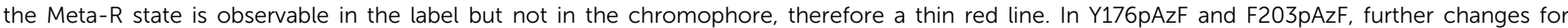

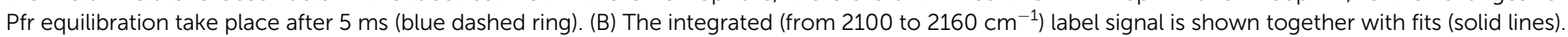
The time constants are presented in Fig. 2-4C. 
F203 is a hydrophobic residue unable to form H-bonds to the side chain and thus differs from pAzF. F203 offers a hydrophobic pocket for the D-ring during the photocycle. The time-resolved IR spectra of the fingerprint region underline the differences between the native residue and pAzF. The importance of phenylalanine in this position for the formation of the Meta-R intermediate becomes apparent, as the label in this position disables the formation of the conventional Meta-R state (Fig. 4E and Fig. S12, ESI $\dagger$ ). As an explanation for this finding we offer the following. In Pfr, the positive signal at $1685 \mathrm{~cm}^{-1}$ originates from the interaction between the BV D-ring carbonyl and $\mathrm{H}_{201 .}{ }^{5}$ In Meta-R of the WT, the BV D-ring is already in its Pfr orientation, but H201 has not yet flipped to form the interaction because F203 is hindering its movement. As a result, the lack of an $\mathrm{H}$-bond to the D-ring carbonyl causes the upshift to $1730 \mathrm{~cm}^{-1}$ in Meta-R. ${ }^{5,45}$ As the azido group is added to F203, the natively hydrophobic residue gains polarity and hence, F203pAzF does not hinder the H201 movement in the transition towards Pfr. In conclusion, the pAzF label at F203 suppresses an intermediate in the formation of the new protein-chromophore interactions of Pfr, which explains why the global analyses of both flash photolysis and time-resolved FTIR data reveal only two components for the photocycle.

\subsection{Time-resolved spectroscopy using IR probes}

FTIR difference spectroscopy has the benefit of yielding information on the global protein mechanism in solution using the fingerprint region while at the same time using the signal around $2100 \mathrm{~cm}^{-1}$ to report on site-specific changes in the label environment. ${ }^{24}$ Azidophenylalanine is superior to other probes due to its high extinction coefficient. Moreover, the shifts of the pAzF freqency reliably report on the H-bonding strength to the label. ${ }^{41}$ However, two other aspects of the interpretation of the pAzF signal are challenging. First, the azide vibrations respond to the (a)polarity of the environment inconsistently and with very minor shifts. ${ }^{40}$ Solvatochromic models of the azidophenyl vibrations have been built, ${ }^{38,41}$ but rather underline the complexity of its influence on the vibrational frequency, and work better for nitrile probes. ${ }^{60-62}$ Secondly, the pAzF signal is accompanied by Fermi resonance bands, which complicate the lineshape and thus the interpretation of the pAzF signal. ${ }^{41,47}$ This complication is exemplified by the interpretation of the Pfr signal of the F203pAzF mutant (Fig. 4B). Lipkin et $a l^{63}$ managed to considerably reduce the Fermi resonances by isotope labeling of the middle and terminal nitrogen of the azido group. Zhang et $a l^{47}$ proposed instead to modulate the Fermi resonances by changing the azide position from para to meta. In contrast, the Fermi resonance bands of azido probes might be utilized to yield further information. In cyano probes, the underlying coupling constant of the Fermi resonances is an indicator of the H-bonding status of the CN-group. ${ }^{64}$ Probably, the H-bonding status of the azido probe effects the Fermi resonance bands as well. Our findings might motivate further studies on the understanding of the Fermi resonances of azide signals in different environments, as it has been progressed for many other vibrational modes, ${ }^{65}$ to utilize the full potential of the azido probes.

The azide vibrational frequency is tuned by the H-bonding strength of the microenvironment, ${ }^{40,41}$ which allows for the detection of structural and conformational changes. Here, we mainly concentrate on residues that have a phenyl ring in the side chain and act as key residues in the photocycle. For example, F203pAzF indicates the importance of the native residue while providing site-specific information on the photoconversion process. However, other residues are also accessible, as demonstrated successfully for R228pAzF (Fig. S5A, ESI $\dagger$ ). Therefore, in future trials the selection of positions should be extended to sites expected to be close to the action but without a key role in the photoconversion, as demonstrated by the Hamm group. ${ }^{29}$ As the pAzF label has proven itself as a successful tool for the investigation of site-specific changes in a time-resolved manner, the label will be applied to the dimeric interface as well as to output domain to study the time scales of signal transduction.

To conclude, by utilizing the vibrational probe pAzF inserted into a phytochrome we obtained the site-selective signal propagation by means of both steady-state and time-resolved IR spectroscopy in the micro- to millisecond time scale. To some extent a concerted signal propagation between the protein and the chromophore is observed, in particular in the tightly linked tongue region. In contrast, other sites nearby the chromophore show individual time scales (dimensions), as reflected in extended equilibration times with respect to those of the chromophore, or in a lack of particular intermediate states. Our results demonstrate that $\mathrm{pAzF}$ is a reliable probe for time-resolved IR spectroscopy, and this approach opens a door to study protein movements in real time at ambient conditions and in solution.

\section{Conflicts of interest}

There are no conflicts to declare.

\section{Acknowledgements}

We thank Peter Hamm and Olga Bozovic for important discussions regarding IR labels, Valentyna Kuznetsova for the help with the data-analysis program, and Rahul Nanekar for preliminary FTIR experiments. The original plasmids for this work were a kind gift of Richard Vierstra and Katrina Forest (pET21b) and Peter Schultz (pEVOL-pAzF). We thank Prof. Thomas O'Haver for the script of the interactive peak fitter. The work of BS-B has been supported by a grant from the Swiss National Science Foundation (P2ZHP2_164991). JAI acknowledges the Academy of Finland (296135 and 332742) and the Jane and Aatos Erkko foundation. HT acknowledges the Academy of Finland (285461 and 330678). LS acknowledges a fellowship of the Studienstiftung des Deutschen Volkes. TK acknowledges a Heisenberg fellowship of the Deutsche Forschungsgemeinschaft (KO3580/4-2). 


\section{Notes and references}

1 N. C. Rockwell, Y.-S. Su and J. C. Lagarias, Annu. Rev. Plant Biol., 2006, 57, 837-858.

2 E. S. Burgie, T. Wang, A. N. Bussell, J. M. Walker, H. Li and R. D. Vierstra, J. Biol. Chem., 2014, 289, 24573-24587.

3 R. A. Sharrock, Genome Biol., 2008, 9, 230.

4 A. Björling, O. Berntsson, H. Lehtivuori, H. Takala, A. J. Hughes, M. Panman, M. Hoernke, S. Niebling, L. Henry, R. Henning, I. Kosheleva, V. Chukharev, N. V. Tkachenko, A. Menzel, G. Newby, D. Khakhulin, M. Wulff, J. A. Ihalainen and S. Westenhoff, Sci. Adv., 2016, 2, e1600920.

5 J. A. Ihalainen, E. Gustavsson, L. Schroeder, S. Donnini, H. Lehtivuori, L. Isaksson, C. Thöing, V. Modi, O. Berntsson, B. Stucki-Buchli, A. Liukkonen, H. Häkkänen, E. Kalenius, S. Westenhoff and T. Kottke, J. Am. Chem. Soc., 2018, 140, 12396-12404.

6 J. R. Wagner, J. S. Brunzelle, K. T. Forest and R. D. Vierstra, Nature, 2005, 438, 325-331.

7 E. S. Burgie, J. Zhang and R. D. Vierstra, Structure, 2016, 24, 448-457.

8 J. J. van Thor, K. L. Ronayne and M. Towrie, J. Am. Chem. Soc., 2007, 129, 126-132.

9 Y. Yang, M. Linke, T. Von Haimberger, J. Hahn, R. Matute, L. González, P. Schmieder and K. Heyne, J. Am. Chem. Soc., 2012, 134, 1408-1411.

10 E. Claesson, W. Y. Wahlgren, H. Takala, S. Pandey, L. Castillon, V. Kuznetsova, L. Henry, M. Panman, M. Carrillo, J. Kübel, R. Nanekar, L. Isaksson, A. Nimmrich, A. Cellini, D. Morozov, M. Maj, M. Kurttila, R. Bosman, E. Nango, R. Tanaka, T. Tanaka, L. Fangjia, S. Iwata, S. Owada, K. Moffat, G. Groenhof, E. A. Stojković, J. A. Ihalainen, M. Schmidt and S. Westenhoff, eLife, 2020, 9, e53514.

11 H. Takala, A. Björling, O. Berntsson, H. Lehtivuori, S. Niebling, M. Hoernke, I. Kosheleva, R. Henning, A. Menzel, J. A. Ihalainen and S. Westenhoff, Nature, 2014, 509, 245-248.

12 C. Song, G. Psakis, C. Lang, J. Mailliet, W. Gärtner, J. Hughes, J. Matysik and C. J. Lagarias, Proc. Natl. Acad. Sci. U. S. A., 2011, 108, 3842-3847.

13 C. Song, T. Rohmer, M. Tiersch, J. Zaanen, J. Hughes and J. Matysik, Photochem. Photobiol., 2013, 89, 259-273.

14 H. Foerstendorf, C. Benda, W. Gärtner, M. Storf, H. Scheer and F. Siebert, Biochemistry, 2001, 40, 14952-14959.

15 J. J. Van Thor, N. Fisher and P. R. Rich, J. Phys. Chem. B, 2005, 109, 20597-20604.

16 N. Lenngren, P. Edlund, H. Takala, B. Stucki-Buchli, J. Rumfeldt, I. Peshev, H. Häkkänen, S. Westenhoff and J. A. Ihalainen, Phys. Chem. Chem. Phys., 2018, 20, 18216-18225.

17 H. Takala, S. Niebling, O. Berntsson, A. Björling, H. Lehtivuori, H. Häkkänen, M. Panman, E. Gustavsson, M. Hoernke, G. Newby, F. Zontone, M. Wulff, A. Menzel, J. A. Ihalainen and S. Westenhoff, Struct. Dyn., 2016, 3, 12.
18 A. Takiden, F. Velazquez-Escobar, J. Dragelj, A. L. Woelke, E.-W. Knapp, P. Piwowarski, F. Bart, P. Hildebrandt and M. A. Mroginski, Photochem. Photobiol., 2017, 93, 713-723.

19 S. Ye, T. Huber, R. Vogel and T. P. Sakmar, Nat. Chem. Biol., 2009, 5, 397-399.

20 A. Creon, I. Josts, S. Niebling, N. Huse and H. Tidow, Struct. Dyn., 2018, 5, 12.

21 K. L. Koziol, P. J. M. Johnson, B. Stucki-Buchli, S. A. Waldauer and P. Hamm, Curr. Opin. Struct. Biol., 2015, 34, 1-6.

22 J. Ma, I. M. Pazos, W. Zhang, R. M. Culik and F. Gai, Annu. Rev. Phys. Chem., 2015, 66, 357-377.

23 H. Kim and M. Cho, Chem. Rev., 2013, 113, 5817-5847.

24 L. Blankenburg, L. Schroeder, F. Habenstein, B. Błasiak, T. Kottke and J. Bredenbeck, Phys. Chem. Chem. Phys., 2019, 21, 6622-6634.

25 K.-I. Oh, J.-H. Lee, C. Joo, H. Han and M. Cho, J. Phys. Chem., 2008, 112, 10352-10357.

26 C. C. Liu and P. G. Schultz, Annu. Rev. Biochem., 2010, 79, 413-444.

27 H. Taskent-Sezgin, J. Chung, P. S. Banerjee, S. Nagarajan, R. B. Dyer, I. Carrico and D. P. Raleigh, Angew. Chem., Int. Ed., 2010, 49, 7473-7475.

28 C. G. Bazewicz, M. T. Liskov, K. J. Hines and S. H. Brewer, J. Phys. Chem. B, 2013, 117, 8987-8993.

29 B. Stucki-Buchli, P. J. M. Johnson, O. Bozovic, C. Zanobini, K. L. Koziol, P. Hamm, A. Gulzar, S. Wolf, S. Buchenberg and G. Stock, J. Phys. Chem. A, 2017, 121, 9435-9445.

30 C. R. Hall, J. Tolentino Collado, J. N. Iuliano, A. A. Gil, K. Adamczyk, A. Lukacs, G. M. Greetham, I. Sazanovich, P. J. Tonge and S. R. Meech, J. Phys. Chem. B, 2019, 123, 9592-9597.

31 B. S. Krause, J. C. D. Kaufmann, J. Kuhne, J. Vierock, T. Huber, T. P. Sakmar, K. Gerwert, F. J. Bartl and P. Hegemann, Biochemistry, 2015, 58, 1275-1286.

32 S. Ye, E. Zaitseva, G. Caltabiano, G. F. X. Schertler, T. P. Sakmar, X. Deupi and R. Vogel, Nature, 2010, 464, 1386-1389.

33 M. C. Thielges, J. Y. Axup, D. Wong, H. S. Lee, J. K. Chung, P. G. Schultz and M. D. Fayr, J. Phys. Chem. B, 2011, 115, 11294-11304.

34 C. Zanobini, O. Bozovic, B. Jankovic, K. L. Koziol, P. J. M. Johnson, P. Hamm, A. Gulzar, S. Wolf and G. Stock, J. Phys. Chem. B, 2018, 122, 10118-10125.

35 J. W. Chin, S. W. Santoro, A. B. Martin, D. S. King, L. Wang and P. G. Schultz, J. Am. Chem. Soc., 2002, 124, 9026-9027.

36 T. S. Young, I. Ahmad, J. A. Yin and P. G. Schultz, J. Mol. Biol., 2010, 395, 361-374.

37 R. Adhikary, J. Zimmermann and F. E. Romesberg, Chem. Rev., 2017, 117, 1927-1969.

38 J. H. Choi, K. I. Oh and M. Cho, J. Chem. Phys., 2008, 129, 174512.

39 X. S. Gai, B. A. Coutifaris, S. H. Brewer and E. E. Fenlon, Phys. Chem. Chem. Phys., 2011, 13, 5926-5930.

40 M. Maj, C. Ahn, B. Błasiak, K. Kwak, H. Han and M. Cho, J. Phys. Chem. B, 2016, 120, 10167-10180. 
41 M. P. Wolfshorndl, R. Baskin, I. Dhawan and C. H. Londergan, J. Phys. Chem. B, 2012, 116, 1172-1179.

42 W. Uhmann, A. Becker, C. Taran and F. Siebert, Appl. Spectrosc., 1991, 45, 390-397.

43 M. E. Auldridge, K. A. Satyshur, D. M. Anstrom and K. T. Forest, J. Biol. Chem., 2012, 287, 7000-7009.

44 H. Takala, A. Björling, M. Linna, S. Westenhoff and J. A. Ihalainen, J. Biol. Chem., 2015, 290, 16383-16392.

45 H. Takala, H. K. Lehtivuori, O. Berntsson, A. Hughes, R. Nanekar, S. Niebling, M. Panman, L. Henry, A. Menzel, S. Westenhoff and J. A. Ihalainen, J. Biol. Chem., 2018, 293, 8161-8172.

46 M. M. Waegele, R. M. Culik and F. Gai, J. Phys. Chem. Lett., 2011, 2, 2598-2609.

47 J. Zhang, L. Wang, J. Zhang, J. Zhu, X. Pan, Z. Cui, J. Wang, W. Fang and Y. Li, J. Phys. Chem. B, 2018, 122, 8122-8133.

48 J. J. Snellenburg, S. P. Laptenok, R. Seger, K. M. Mullen and I. H. M. van Stokkum, J. Stat. Softw., 2012, 49, 1-22.

49 E. Gustavsson, L. Isaksson, C. Persson, M. Mayzel, U. Brath, L. Vrhovac, J. A. Ihalainen, G. Karlsson, V. Orekhov and S. Westenhoff, Biophys. J., 2020, 118, 415-421.

50 Q.-Z. Xu, L. Goett-Zink, W. Gärtner, K.-H. Zhao and T. Kottke, Biochemistry, 2020, 59, 2047-2054.

51 J. Kübel, M. Chenchiliyan, S. A. Ooi, E. Gustavsson, L. Isaksson, V. Kuznetsova, J. A. Ihalainen, S. Westenhoff and M. Maj, Phys. Chem. Chem. Phys., 2020, 22, 9195-9203.

52 I. H. van Stokkum, D. S. Larsen and R. van Grondelle, Biochim. Biophys. Acta, Bioenerg., 2004, 1657, 82-104.

53 T. Kottke, V. A. Lórenz-Fonfría and J. Heberle, J. Phys. Chem. $B, 2017,121,335-350$.
54 J. R. Wagner, J. Zhang, D. von Stetten, M. Günther, D. H. Murgida, M. A. Mroginski, J. M. Walker, K. T. Forest, P. Hildebrandt and R. D. Vierstra, J. Biol. Chem., 2008, 283, 12212-12226.

55 A. Barth, Biochim. Biophys. Acta, Bioenerg., 2007, 1767, 1073-1101.

56 R. Takahashi, K. Okajima, H. Suzuki, H. Nakamura, M. Ikeuchi and T. Noguchi, Biochemistry, 2007, 46, 6459-6467.

57 J. J. Van Thor, B. Borucki, W. Crielaard, H. Otto, T. Lamparter, J. Hughes, K. J. Hellingwerf and M. P. Heyn, Biochemistry, 2001, 40, 11460-11471.

58 B. Borucki, D. Von Stetten, S. Seibeck, T. Lamparter, N. Michael, M. A. Mroginski, H. Otto, D. H. Murgida, M. P. Heyn and P. Hildebrandt, J. Biol. Chem., 2005, 280, 34358-34364.

59 H. Takala, P. Edlund, S. Ihalainen and J. A. Westenhoff, Photochem. Photobiol. Sci., 2020, 19, 1488-1510.

60 S. Bagchi, S. D. Fried and S. G. Boxer, J. Am. Chem. Soc., 2012, 134, 10373-10376.

61 L. J. G. W. van Wilderen, D. Kern-Michle, H. MüllerWerkmeister and J. Bredenbeck, Phys. Chem. Chem. Phys, 2014, 16, 19643-19653.

62 W. Zhang, B. N. Markiewicz, R. S. Doerkse, A. B. Smith and F. Gai, Phys. Chem. Chem. Phys., 2016, 18, 7027-7034.

63 J. S. Lipkin, R. Song, E. E. Fenlon and S. H. Brewer, J. Phys. Chem. Lett., 2011, 2, 1672-1676.

64 J. M. Rodgers, R. M. Abaskharon, B. Ding, J. Chen, W. Zhang and F. Gai, Phys. Chem. Chem. Phys., 2017, 19, 16144-16150.

65 S. D. Fried and S. G. Boxer, Acc. Chem. Res., 2015, 48, 998-1006. 